\title{
Orthogonal Engineering of Biosynthetic Pathway for Efficient Production of Limonene in Saccharomyces cerevisiae
}

Si Cheng ${ }^{\dagger}$, Xue Liu ${ }^{\dagger}$, Guozhen Jiang ${ }^{\dagger}$, Jihua $\mathrm{Wu}^{\dagger}$, Dengwei Lei ${ }^{\dagger}$, Jin-lai Zhang ${ }^{\dagger}$, Ying-Jin Yuan ${ }^{\dagger, \ddagger}$ Jianjun Qiao ${ }^{\dagger, \dagger}$, and Guang-Rong Zhao ${ }^{*}, \dagger, \ddagger$

${ }^{\dagger}$ Frontier Science Center for Synthetic Biology and Key Laboratory of Systems Bioengineering (Ministry of Education), School of Chemical Engineering and Technology, Tianjin University, Yaguan Road 135, Jinnan District, Tianjin 300350, China

\$ SynBio Research Platform, Collaborative Innovation Centre of Chemical Science and Engineering (Tianjin), Tianjin University, Yaguan Road 135, Jinnan District, Tianjin 300350, China

*Corresponding author: grzhao@tju.edu.cn

Tel: +86-22-85356580; Fax: +86-22-27403389 
Table S1: Strains and plasmids used in this study.

Table S2: Primers used in this study.

Table S3: Codon-optimized sequences of truncated LSs and NPPS in this study.

Fig. S1: Alignment of amino acid sequences of nine limonene synthases.

Fig. S2: Phylogenetic analysis of nine LSs proteins.

Fig. S3: Biomass of limonene-producing strains harboring the CLB pathway at $48 \mathrm{~h}$.

Fig. S4: Biomass of limonene-producing strains harboring the OLB pathway at $48 \mathrm{~h}$.

Fig. S5: Titer of limonene in strain LimY28 feeding with glucose and ethanol at $20 \mathrm{~h}$, respectively. 
Table S1

Strains and plasmids used in this study

\begin{tabular}{|c|c|c|}
\hline Name & Discription & Source \\
\hline \multicolumn{3}{|l|}{ Strains } \\
\hline yJGZ1 & 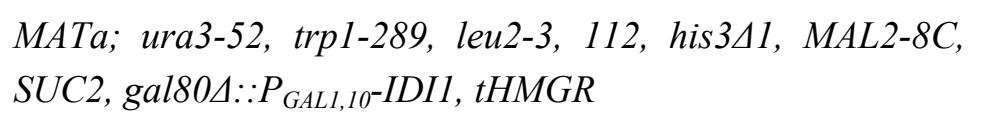 & 7 \\
\hline LimY1 & yJGZ1 with pCS01 & This study \\
\hline LimY2 & yJGZ1 with pCS02 & This study \\
\hline LimY3 & yJGZ1 with pCS03 & This study \\
\hline LimY4 & yJGZ1, pCS04 & This study \\
\hline LimY5 & yJGZ1, pCS05 & This study \\
\hline LimY6 & yJGZ1, pCS06 & This study \\
\hline $\operatorname{LimY7}$ & yJGZ1, pCS07 & This study \\
\hline $\operatorname{LimY8}$ & yJGZ1, pCS08 & This study \\
\hline LimY9 & yJGZ1, pCS09 & This study \\
\hline LimY10 & yJGZ1, pCS10 & This study \\
\hline LimY11 & yJGZ1, pCS11 & This study \\
\hline LimY12 & yJGZ1, pCS12 & This study \\
\hline LimY13 & yJGZ1, pCS13 & This study \\
\hline LimY14 & yJGZ1, pCS14 & This study \\
\hline LimY15 & yJGZ1, pCS15 & This study \\
\hline LimY16 & yJGZ1, pCS16 & This study \\
\hline LimY17 & yJGZ1, pCS17 & This study \\
\hline LimY18 & yJGZ1, pCS18 & This study \\
\hline yCS1 & $\mathrm{yJGZ1}, P_{E R G 20} \triangle: \because P_{H X T 1}$ & This study \\
\hline LimY26 & $\mathrm{yCS} 1, \mathrm{pCS} 3$ & This study \\
\hline LimY28 & yCS1, pCS13 & This study \\
\hline \multicolumn{3}{|l|}{ Plasmids } \\
\hline pRS415K & $\begin{array}{l}\text { substitution of kanamycin resistant gene for ampicillin } \\
\text { resistant gene based on pRS415 }\end{array}$ & 7 \\
\hline pZJL1 & $\mathrm{pRS} 415 \mathrm{~K}-\mathrm{T}_{E N O 2}-\mathrm{P}_{G A L 10}-\mathrm{T}_{G P M I}$ & This study \\
\hline pJGZ1 & $\mathrm{pRS} 415 \mathrm{~K}-\mathrm{T}_{G P M I}-\mathrm{P}_{G A L 7}-\mathrm{T}_{G P D}$ & 7 \\
\hline pJGZ38 & $\mathrm{pRS} 415 \mathrm{~K}-\mathrm{T}_{G P M I}-\mathrm{P}_{G A L 7}-E R G 20^{W W}-t 3 C r G E S-\mathrm{T}_{G P D}$ & 7 \\
\hline pCSL1 & $\mathrm{pRS} 415 \mathrm{~K}-\mathrm{T}_{G P M 1^{-}}-\mathrm{P}_{G A L 7^{-}} C u t L S 1-\mathrm{T}_{G P D}$ & This study \\
\hline pCSL2 & $\mathrm{pRS} 415 \mathrm{~K}-\mathrm{T}_{G P M I^{-}}-\mathrm{P}_{G A L T^{-}} C u t L S 2-\mathrm{T}_{G P D}$ & This study \\
\hline pCSL3 & $\mathrm{pRS} 415 \mathrm{~K}-\mathrm{T}_{G P M I^{-}}-\mathrm{P}_{G A L T^{-}} C l t L S 1-\mathrm{T}_{G P D}$ & This study \\
\hline pCSL4 & $\mathrm{pRS} 415 \mathrm{~K}-\mathrm{T}_{G P M I}-\mathrm{P}_{G A L T^{-}} C l t L S 2-\mathrm{T}_{G P D}$ & This study \\
\hline pCSL5 & $\mathrm{pRS} 415 \mathrm{~K}-\mathrm{T}_{G P M 1}-\mathrm{P}_{G A L 7^{-}} C l t L S 3-\mathrm{T}_{G P D}$ & This study \\
\hline pCSL6 & $\mathrm{pRS} 415 \mathrm{~K}-\mathrm{T}_{G P M I}-\mathrm{P}_{G A L T}-C s t L S-\mathrm{T}_{G P D}$ & This study \\
\hline pCSL7 & $\mathrm{pRS} 415 \mathrm{~K}-\mathrm{T}_{G P M I}-\mathrm{P}_{G A L T}-P t t L S-\mathrm{T}_{G P D}$ & This study \\
\hline pCSL8 & $\mathrm{pRS} 415 \mathrm{~K}-\mathrm{T}_{G P M I}-\mathrm{P}_{G A L 7}-S h t L S-\mathrm{T}_{G P D}$ & This study \\
\hline pCSL9 & $\mathrm{pRS} 415 \mathrm{~K}-\mathrm{T}_{G P M I}-\mathrm{P}_{G A L T}-A r t L S-\mathrm{T}_{G P D}$ & This study \\
\hline pCSG1 & $\mathrm{pRS} 415 \mathrm{~K}-\mathrm{T}_{E N O 2}-\mathrm{P}_{G A L 10}-E R G 20^{W W}-\mathrm{T}_{G P M I}$ & This study \\
\hline pCSN1 & $\mathrm{pRS} 415 \mathrm{~K}-\mathrm{T}_{E N O 2^{-}}-\mathrm{P}_{G A L 10^{-}} N D P S 1-\mathrm{T}_{G P M 1}$ & This study \\
\hline pCS & pRS426 possessing $\mathrm{T}_{E N O 2}-\mathrm{T}_{G P D}$ & This study \\
\hline
\end{tabular}




\begin{tabular}{|c|c|c|c|}
\hline pCS01 & $\begin{array}{l}\text { pRS426 possessing } \\
\text { CutLS1-T } \mathrm{T}_{G P D}\end{array}$ & $\mathrm{~T}_{E N O 2}-\mathrm{P}_{G A L 10^{-}} E R G 20^{W W}-\mathrm{T}_{G P M 1}-\mathrm{P}_{G A L 7^{-}}$ & This study \\
\hline pCS02 & $\begin{array}{l}\text { pRS426 possessing } \\
\text { CutLS2-T } \mathrm{T}_{G P D}\end{array}$ & $\mathrm{~T}_{E N O 2^{-}}-\mathrm{P}_{G A L 10^{-}} E R G 20^{W W}-\mathrm{T}_{G P M 1^{-}}-\mathrm{P}_{G A L 7^{-}}$ & This study \\
\hline pCS03 & $\begin{array}{l}\text { pRS426 possessing } \\
\text { CltLS1- } \mathrm{T}_{G P D}\end{array}$ & $\mathrm{~T}_{E N O 2^{-}}-\mathrm{P}_{G A L 10^{-}}-E R G 20^{W W}-\mathrm{T}_{G P M 1^{-}}-\mathrm{P}_{G A L 7^{-}}$ & This study \\
\hline pCS04 & $\begin{array}{l}\text { pRS426 possessing } \\
C l t L S 2-\mathrm{T}_{G P D}\end{array}$ & $\mathrm{~T}_{E N O 2^{-}}-\mathrm{P}_{G A L 10^{-}} E R G 20^{W W}-\mathrm{T}_{G P M 1^{-}}-\mathrm{P}_{G A L 7^{-}}$ & This study \\
\hline pCS05 & $\begin{array}{l}\text { pRS426 possessing } \\
C l t L S 3-\mathrm{T}_{G P D}\end{array}$ & $\mathrm{~T}_{E N O 2^{-}}-\mathrm{P}_{G A L 10^{-}}-E R G 20^{W W}-\mathrm{T}_{G P M 1^{-}}-\mathrm{P}_{G A L 7^{-}}$ & This study \\
\hline pCS06 & $\begin{array}{l}\mathrm{pRS} 426 \text { possessing } \\
\text { Cst } L S-\mathrm{T}_{G P D}\end{array}$ & $\mathrm{~T}_{E N O 2^{-}}-\mathrm{P}_{G A L 10^{-}} E R G 20^{W W}-\mathrm{T}_{G P M 1^{-}}-\mathrm{P}_{G A L 7^{-}}$ & This study \\
\hline pCS07 & $\begin{array}{l}\text { pRS426 possessing } \\
P t t L S-\mathrm{T}_{G P D}\end{array}$ & $\mathrm{~T}_{E N O 2-}-\mathrm{P}_{G A L 10^{-}} E R G 20^{W W}-\mathrm{T}_{G P M 1}-\mathrm{P}_{G A L 7^{-}}$ & This study \\
\hline pCS08 & $\begin{array}{l}\text { pRS426 possessing } \\
\text { ShtLS-T } \mathrm{T}_{G P D}\end{array}$ & $\mathrm{~T}_{E N O 2^{-}}-\mathrm{P}_{G A L 10^{-}} E R G 20^{W W}-\mathrm{T}_{G P M 1^{-}}-\mathrm{P}_{G A L 7^{-}}$ & This study \\
\hline pCS09 & $\begin{array}{l}\mathrm{pRS} 426 \text { possessing } \\
\text { ArtLS-T } \mathrm{T}_{G P D}\end{array}$ & $\mathrm{~T}_{E N O 2^{-}}-\mathrm{P}_{G A L 10^{-}}-E R G 20^{W W}-\mathrm{T}_{G P M 1^{-}}-\mathrm{P}_{G A L 7^{-}}$ & This study \\
\hline pCS10 & $\begin{array}{l}\text { pRS426 possessing } \mathrm{T}_{E N O} \\
\mathrm{~T}_{G P D}\end{array}$ & ${ }_{22}-\mathrm{P}_{G A L 10}-N D P S 1-\mathrm{T}_{G P M I}-\mathrm{P}_{G A L 7}-C u t L S 1-$ & This study \\
\hline pCS11 & 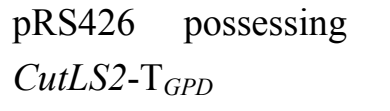 & $\mathrm{T}_{E N O 2^{-}}-\mathrm{P}_{G A L 10^{-}} \quad N D P S 1-\mathrm{T}_{G P M 1}-\mathrm{P}_{G A L 7^{-}}$ & This study \\
\hline pCS12 & $\begin{array}{l}\mathrm{pRS} 426 \text { possessing } \mathrm{T}_{E N C} \\
-\mathrm{T}_{G P D}\end{array}$ & ${ }_{02}-\mathrm{P}_{G A L 10^{-}}$NDPS1- $\mathrm{T}_{G P M 1^{-}}-\mathrm{P}_{G A L 7^{-}} C l t L S 1$ & This study \\
\hline pCS13 & $\begin{array}{l}\text { pRS426 possessing } \mathrm{T}_{E N C} \\
-\mathrm{T}_{G P D}\end{array}$ & ${ }_{02}-\mathrm{P}_{G A L 10^{-}}$NDPS1- $\mathrm{T}_{G P M 1^{-}}-\mathrm{P}_{G A L 7^{-}} C l t L S 2$ & This study \\
\hline pCS14 & $\begin{array}{l}\mathrm{pRS} 426 \text { possessing } \mathrm{T}_{E N C} \\
-\mathrm{T}_{G P D}\end{array}$ & ${ }_{02}-\mathrm{P}_{G A L 10^{-}} N D P S 1-\mathrm{T}_{G P M 1}-\mathrm{P}_{G A L 7}-C l t L S 3$ & This study \\
\hline pCS15 & $\begin{array}{l}\text { pRS426 possessing } \mathrm{T}_{E N O} \\
\mathrm{~T}_{G P D}\end{array}$ & ${ }_{02}-\mathrm{P}_{G A L 10^{-}}$NDPS1- $\mathrm{T}_{G P M 1}-\mathrm{P}_{G A L T^{-}} C s t L S-$ & This study \\
\hline pCS16 & $\begin{array}{l}\text { pRS426 possessing } \mathrm{T}_{E N O} \\
\mathrm{~T}_{G P D}\end{array}$ & ${ }_{22}-\mathrm{P}_{G A L 10^{-}}$NDPS1- $\mathrm{T}_{G P M 1}-\mathrm{P}_{G A L 7^{-}} P t t L S-$ & This study \\
\hline pCS17 & $\begin{array}{l}\mathrm{pRS} 426 \text { possessing } \mathrm{T}_{E N O} \\
\mathrm{~T}_{G P D}\end{array}$ & ${ }_{22}-\mathrm{P}_{G A L 10^{-}}$NDPS1- $\mathrm{T}_{G P M 1}-\mathrm{P}_{G A L 7^{-}}$ShtLS - & This study \\
\hline pCS18 & $\begin{array}{l}\mathrm{pRS} 426 \text { possessing } \mathrm{T}_{E N O} \\
\mathrm{~T}_{G P D}\end{array}$ & ${ }_{22}-\mathrm{P}_{G A L 10^{-}} N D P S 1-\mathrm{T}_{G P M I}-\mathrm{P}_{G A L 7^{-}}$ArtLS - & This study \\
\hline
\end{tabular}




\section{Table S2}

Primers used in this study

\begin{tabular}{|c|c|}
\hline Primer & Sequence \\
\hline ERG20ww-BsaI-F & GGTCTCAATGGCTTCAGAAAAAGAAATTAGG \\
\hline ERG20ww-BsaI-R & GGTCTCTTTACTATTTGCTTCTCTTGTAAACT \\
\hline SINDPS1-BsaI-F & GGTCTCAATGTCTGCTAGAGGTTTGAACA \\
\hline SINDPS1-BsaI-R & GGTCTCTTTAGTAAGTGTGACCACCGAAT \\
\hline CutLS1-BsaI-F & GGTCTCAATGGATAGAAGATCTGCTAATTACC \\
\hline CutLS1-BsaI-R & GGTCTCTTTAACCTTTTGTACCTGGAGAAG \\
\hline CutLS2-BsaI-F & GGTCTCAATGGATAGAAGATCAGCAAATTAC \\
\hline CutLS2-BsaI-R & GGTCTCTTTAACCTTTTGTAACTGGTGAAGAA \\
\hline CltLS1-BsaI-F & GGTCTCAATGGATAGAAGATCTGCTAATTACC \\
\hline CltLS1-BsaI-R & GGTCTCTTTAACCTTTAGTACCTGGAGAAGCA \\
\hline CltLS2-BsaI-F & GGTCTCAATGGATAGAAGATCTGCTAATTATC \\
\hline CltLS2-BsaI-R & GGTCTCTTTAACCTTTAGTACCTGGAGAAGCA \\
\hline CltLS3-BsaI-F & GGTCTCAATGATCAGAAGATCTGCTGATTAT \\
\hline CltLS3-BsaI-R & GGTCTCTTTATGGAATTGGATCAATAAACAAA \\
\hline CstLS-BsaI-F & GGTCTCAATGGATAGAAGATCCGCTAATT \\
\hline CstLS-BsaI-R & GGTCTCTTTAACCCTTAGTACCTGGAGAAGC \\
\hline PttLS-BsaI-F & GGTCTCAATGGATAGAAGATCTGCTAATTACC \\
\hline PttLS-BsaI-R & GGTCTCTTTAACCTTTTGTAACTGGTGAAGA \\
\hline ShtLS-BsaI-F & GGTCTCAATGTGCAGCCACAGTACCA \\
\hline ShtLS-BsaI-R & GGTCTCTTTAATGATTGAGTGGTTTGTAAAAGACTTC \\
\hline ArtLS-BsaI-F & GGTCTCAATGGAAAGAAGATCGGGTAACTA \\
\hline ArtLS-BsaI-R & GGTCTCTTTAAGCGAATGGTTGGAACAAAC \\
\hline ENO2t-F & GGGAGATGGAGATGATACCT \\
\hline GPM1t-R & TATTCGAACTGCCCATTCAGCT \\
\hline GPM1t-F & TGCACCAATTGCAAAGGGAAAA \\
\hline GPDt-R & GCAAGATTTAAAGTAAATTCAC \\
\hline ENO2t-NotI-F & GCGGCCGCAGTGCTTTTAACTAAGAATTATTAGTC \\
\hline ENO2t-BglII-R & AGATCTAGGTATCATCTCCATCTCCCATAT \\
\hline GPDt- BglII-F & AGATCTGTGAATTTACTTTAAATCTTGCATTT \\
\hline GPDt- NotI-R & AAGGAAAAAAGCGGCCGCGGAATCTGTGTAT \\
\hline $\mathrm{P}_{E R G 20^{-}-\mathrm{up}-\mathrm{F}}$ & GACAATCATTACCACAAGATGAACAC \\
\hline $\mathrm{P}_{E R G 20^{-} \text {down-F }}$ & ATGGCTTCAGAAAAAGAAATTAGGAGA \\
\hline $\mathrm{P}_{E R G 20^{-}-\mathrm{up}-\mathrm{R}}$ & ACCGCTTAGAATACCTCACACTG \\
\hline $\mathrm{P}_{E R G 20^{-} \text {down-R }}$ & GTCGACTTTGTCTTCAGGTGC \\
\hline $\mathrm{P}_{H X T 1}-\mathrm{F}$ & TGCAGGTCTCATCTGGAATATAATTCC \\
\hline $\mathrm{P}_{H X T 1}-\mathrm{R}$ & GATTTTACGTATATCAACTAGTTGACGATTATG \\
\hline $\mathrm{P}_{t r p}-\mathrm{F}$ & AACGACATTACTATATATATAATATAGGAAGCAT \\
\hline $\mathrm{P}_{t r p}-\mathrm{R}$ & CTATTTCTTAGCATTTTTGACGAAATTTGC \\
\hline $\mathrm{UP}-\mathrm{P}_{t r p}-\mathrm{F}$ & $\begin{array}{l}\text { TGTGAGGTATTCTAAGCGGTAACGACATTACTATATA } \\
\text { TATAATATAGGAAGCAT }\end{array}$ \\
\hline
\end{tabular}




\begin{tabular}{ll} 
UP- $\mathrm{P}_{t r p}-\mathrm{R}$ & TCCTATATTATATATATAGTAATGTCGTTACCGCTTAG \\
& AATACCTCACACTG \\
$\mathrm{P}_{t r p}-\mathrm{P}_{H X T 1}-\mathrm{F}$ & CGTCAAAAATGCTAAGAAATAGTGCAGGTCTCATCTG \\
& GAATATAATTCC \\
$\mathrm{P}_{t r p}-\mathrm{P}_{H X T 1}-\mathrm{R}$ & TATATTCCAGATGAGACCTGCACTATTTCTTAGCATTT \\
& TTGACGAAATTTGC \\
$\mathrm{P}_{H X T 1}$-down-F & AACTAGTTGATATACGTAAAATCATGGCTTCAGAAAA \\
& AGAAATTAGGAGA \\
$\mathrm{P}_{H X T 1}$-down-R & TAATTTCTTTTCTGAAGCCATGATTTTACGTATATCA \\
& ACTAGTTGACGATTATG \\
ZH-F & TCACCCAATTCTCATGGTAGCG \\
ZH-R & TCAGAGGCGTTCCAACTGATGA \\
\hline
\end{tabular}

* BsaI, NotI and BglII restriction sites are blue, yellow and green highlighted respectively. 


\section{Table S3}

Codon-optimized sequences of truncated $L S S$ and NPPS in this study

\begin{tabular}{|c|c|}
\hline Gene & Encoding sequences \\
\hline \multirow[t]{35}{*}{ CutLS1 } & ATGGATAGAAGATCTGCTAATTACCAACCATCAATTTGGGATCATGATT \\
\hline & TCTTGCAATCTTTAAATTCTAACTACACTGATGAAACATACAAAAGAA \\
\hline & GAGCAGAAGAATTGAAGGGTAAAGTTAAGACTGCTATTAAAGATGTTA \\
\hline & CAGAACCATTAGATCAATTAGAATTGATTGATAATTTGCAAAGATTAG \\
\hline & GTTTGGCATACCATTTTGAACCAGAAATCAGAAACATCTTGAGAAACA \\
\hline & TCCATAATCATAATAAGGATTATAATTGGAGAAAAGAAAATTTGTACG \\
\hline & CTACTTCTTTGGAGTTTAGATTGTTAAGACAACATGGTTATCCAGTTTC \\
\hline & TCAAGAAGTTTTCTCTGGTTTTAAAGATGATAAAGTTGGTTTTATTTGT \\
\hline & GATGATTTCAAGGGTATTTTATCATTGCATGAAGCATCTTACTACTCAT \\
\hline & TAGAAGGCGAATCTATTATGGAAGAAGCATGGCAATTCACTTCAAAAC \\
\hline & ATTTGAAGGAAATGATGATCACATCTAATTCAAAAGAAGAAGATGTTT \\
\hline & TTGTTGCTGAACAAGCAAAAAGAGCTTTAGAATTGCCATTACATTGGA \\
\hline & AGAAAGTTCCAATGTTGGAAGCTAGATGGTTTATTCATGTTTACGAAA \\
\hline & AGAGAGAAGATAAGAACCATTTGTTATTGGAATTGGCAAAATTGGAGT \\
\hline & TTAATACTTTACAAGCTATCTATCAAGAAGAATTGAAGGATATTTCTGG \\
\hline & TTGGTGGAAAGATACAGGTTTGGGTGAAAAATTATCATTTGCAAGAAA \\
\hline & TAGATTAGTTGCTTCATTTTTGTGGTCAATGGGTATTGCATTTGAACCA \\
\hline & CAATTCGCTTACTGTAGAAGAGTTTTGACTATTTCTATTGCATTGATCA \\
\hline & CAGTTATTGATGATATATATGATGTTTACGGTACTTTGGATGAATTAGA \\
\hline & АATTTTTACAGATGCTGTTGCAAGATGGGATATTAACTACGCTTTGAAG \\
\hline & CATTTGCCAGGTTACATGAAAATGTGTTTCTTGGCATTGTACAACTTCG \\
\hline & TTAACGAATTTGCTTATTACGTTTTAAAACAACAAGATTTTGATATGTT \\
\hline & GTTGTCTATTAAACATGCTTGGTTGGGTTTAATCCAAGCATATTTGGTT \\
\hline & GAGGCTAAGTGGTATCATTCTAAGTACACTCCAAAATTGGAAGAATAC \\
\hline & TTAGAAAACGGTTTGGTTTCAATTACTGGTCCATTGATCATCACAATTT \\
\hline & CTTATTTGTCAGGTACAAACCCAATTATTAAGAAAGAATTGGAATTTTT \\
\hline & AGAATCAAATCCAGATATTGTTCATTGGTCTTCAAAGATTTTTAGATTA \\
\hline & CAAGATGATTTGGGTACTTCTTCAGATGAAATTCAAAGAGGTGACGTT \\
\hline & CCAAAATCTATTCAATGTTACATGCATGAAACAGGTGCATCAGAAGAA \\
\hline & GTTGCTAGAGAACATATCAAGGATATGATGAGACAAATGTGGAAGAA \\
\hline & AGTTAATGCATACACTGCTGATAAAGATTCTCCATTAACAAGAACTAC \\
\hline & AGCTGAATTTTTGTTGAATTTGGTTAGAATGTCACATTTCATGTATTTGC \\
\hline & ATGGTGACGGTCATGGTGTTCAAAACCAAGAAACTATTGATGTTGGTTT \\
\hline & TACATTGTTGTTCCAACCAATCCCATTGGAAGATAAAGATATGGCTTTT \\
\hline & ACTGCTTCTCCAGGTACAAAAGGTTAA \\
\hline \multirow[t]{6}{*}{ CutLS2 } & ATGGATAGAAGATCAGCAAATTACCAACCATCTATTTGGGATCATGAT \\
\hline & TTCTTGCAATCTTTAAATTCTAACTACACTGATGAAACATACAAAAGAA \\
\hline & GAGAAGAAGAATTGAAGGGTAAAGTTATGACTACAATTAAAGATGTTA \\
\hline & CTGAACCATTGAACCAATTAGAATTGATTGATTCATTGCAAAGATTAG \\
\hline & GTTTGGCTTACCATTTCGAAACAGAAATCAGAAACATCTTGCATGATAT \\
\hline & ATATAATTCTAATAATGATTACGTTTGGAGAAAGGAAAATTTGTACGC \\
\hline
\end{tabular}


AACTTCTTTGGAGTTTAGATTGTTAAGACAACATGGTTACCCAGTTTCT CAAGAAGTTTTTAATGGTTTTAAAGATGATCAAGGTGGTTTTATTTGTG ATGATTTCAAGGGTGTTTTATCTTTGCATGAAGCATCTTACTTCTCATTA GAAGGCGAATCAATTATGGAAGAAGCATGGCAATTCACTTCTAAGCAT TTGAAGGAAGTTATGATTTCTAAGTCAAAGCAAGGTGACGTTTTCGTTG CTGAACAAGCAAAAAGAGGTTTAGAATTGCCATTACATTGGAAAGTTC CAATGTTAGAAGCTAGATGGTTCATTGATGTTTACGAAAAGAGAGAAG ATAAGAACCATTTGTTATTGGAATTGGCTAAATTGGAGTTTAATGTTTT ACAAGCAATCTATCAAGAAGAATTGAAGGATGTTTCAAGATGGTGGAA GGATATTGGTTTGGGTGAAAAATTATCTTTTGCTAGAGATTCTTTGGTT GCATCATTTGTTTGGTCTATGGGTATCGTTTTCGAACCACAATTCGCTT ATTGTAGAAGAATTTTAACTATCACATTTGCATTGATTTCTGTTATTGAT GATATATATGATGTTTACGGTACTTTAGATGAATTGGAATTGTTCGCTG ATGCAGTTGAAAGATGGGATATTAACTACGCTTTGAACCATTTGCCAG ATTACATGAAGATCTGTTTCTTGGCATTGTACAATTTGGTTAACGAGTT TACTTATTACGTTTTAAAACAACAAGATTTTGATATTTTGAGATCAATT AAAAATGCTTGGTTGAGAAACATCCAAGCATATTTGGTTGAAGCAAAA TGGTATCATGGTAAATACACTCCAACATTGGGTGAATTTTTAGAAAAC GGTTTGGTTTCAATTGGTGGTCCAATGGTTACTATGACAGCTTATTTGT CTGGTACTAACCCAATCATCGAAAAGGAATTGGAATTTTTAGAATCTA ATCAAGATATTTCTCATTGGTCTTTTAAAATCTTGAGATTACAAGATGA TTTGGGTACTTCTTCAGATGAAATTAGAAGAGGTGACGTTCCAAAATC AATTCAATGTTACATGCATGAAACAGGTGCTTCTGAAGAAGTTGCAAG AGAACATATCAAGGATATGATGAGACAAATGTGGAAGAAAGTTAACG CTTACAGAGCAGATAAGGATTTCCCATTGTCACAAACTACAGTTGAGTT TATTTTGAACGTTGTTAGAGTTTCTCATTTTATGTATTTGCATGGTGACG GTCATGGTGCTCAAAACCAAGAAACTATGGATGTTGTTTTTACATTGTT GTTCCAACCAATTCCATTGGATGATAAACATATTGTTGCAACTTCTTCA CCAGTTACAAAAGGTTAA

CltLS1 ATGGATAGAAGATCTGCTAATTATCAACCATCAATTTGGGATCATGATT TCTTGCAATCTTTAAATTCTAACTACACAGATGAAGCATACAAAAGAA GAGCTGAAGAATTACGTGGTAAAGTTAAGATCGCAATTAAAGATGTTA TTGAACCATTAGATCAATTGGAATTGATTGATAATTTGCAAAGATTGGG TTTAGCTCATAGATTTGAAACTGAAATTAGAAACATCTTGAACAACATC TATAACAACAATAAGGATTACAACTGGAGAAAGGAAAATTTGTACGCT ACTTCTTTGGAGTTTAGATTGTTAAGACAACATGGTTACCCAGTTTCAC AAGAAGTTTTTAATGGTTTTAAAGATGATCAAGGTGGTTTTATTTGTGA TGATTTCAAGGGTATTTTGTCTTTACATGAAGCATCTTACTACTCATTA GAAGGCGAATCAATTATGGAAGAAGCATGGCAATTCACTTCTAAACAT TTGAAGGAAGTTATGATTTCTAAAAATATGGAAGAAGATGTTTTTGTTG CTGAACAAGCAAAAAGAGCTTTGGAATTACCATTGCATTGGAAAGTTC CAATGTTGGAAGCTAGATGGTTCATCCATATCTATGAAAGAAGAGAAG ATAAGAACCATTTGTTATTGGAATTGGCAAAGATGGAGTTTAATACATT ACAAGCTATCTATCAAGAAGAATTGAAGGAAATTTCTGGTTGGTGGAA 
AGATACTGGTTTAGGTGAAAAATTGTCATTTGCAAGAAATAGATTGGT TGCTTCATTTTTGTGGTCAATGGGTATTGCATTTGAACCACAATTTGCTT ACTGTAGAAGAGTTTTGACAATTTCTATTGCATTGATCACTGTTATTGA TGATATATATGATGTTTACGGTACATTAGATGAATTGGAAATTTTTACT GATGCAGTTGAAAGATGGGATATTAACTACGCTTTGAAGCATTTGCCA GGTTACATGAAGATGTGTTTCTTGGCATTGTACAACTTCGTTAACGAAT TTGCTTATTACGTTTTAAAACAACAAGATTTTGATTTGTTGTTATCTATT AAAAATGCTTGGTTGGGTTTGATCCAAGCATATTTGGTTGAGGCTAAGT GGTATCATTCTAAGTACACTCCAAAATTAGAAGAATACTTGGAAAACG GTTTAGTTTCAATTACAGGTCCATTGATCATCACTATTTCTTATTTGTCA GGTACAAACCCAATTATTAAGAAAGAATTAGAATTTTTGGAATCAAAT CCAGATATTGTTCATTGGTCTTCAAAGATTTTTAGATTGCAAGATGATT TGGGTACATCTTCAGATGAAATTCAAAGAGGTGACGTTCCAAAATCTA TTCAATGTTACATGCATGAAACTGGTGCATCAGAAGAAGTTGCTAGAC AACATATCAAGGATATGATGAGACAAATGTGGAAGAAAGTTAATGCAT ACACAGCTGATAAAGATTCTCCATTAACTGGTACTACAACTGAATTTTT GTTGAATTTGGTTAGAATGTCACATTTCATGTATTTGCATGGTGACGGT CATGGTGTTCAAAACCAAGAAACAATTGATGTTGGTTTTACTTTGTTGT TCCAACCAATCCCATTGGAAGATAAGCACATGGCTTTTACTGCTTCTCC AGGTACTAAAGGTTAA

CltLS2 ATGGATAGAAGATCTGCTAATTACCAACCATCAATTTGGGATCATGATT TCTTGCAATCTTTAAATTCTAACTACACAGATGAAACTTACAGAAGAA GAGCAGAAGAATTGAAGGGTAAAGTTAAGATCGCTATTAAAGATGTTA CAGAACCATTGGATCAATTAGAATTGATTGATAATTTGCAAAGATTAG GTTTGGCATATAGATTTGAAACTGAAATTAGAAACATCTTGCATAACAT CTATAACAACAATAAGGATTACGTTTGGAGAAAGGAAAATTTGTACGC TACTTCTTTGGAGTTTAGATTGTTAAGACAACATGGTTACCCAGTTTCA CAAGAAGTTTTTAATGGTTTTAAAGATGATCAAGGTGGTTTTATTTTCG ATGATTTCAAGGGTATTTTATCTTTGCATGAAGCATCTTACTACTCATT GGAAGGCGAATCTATTATGGAAGAAGCATGGCAATTCACTTCAAAGCA TTTGAAGGAAGTTATGATTTCTAAATCAATGGAAGAAGATGTTTTTGTT GCTGAACAAGCAAAAAGAGCTTTAGAATTGCCATTACATTGGAAAGTT CCAATGTTAGAAGCTAGATGGTTTATTCATGTTTACGAAAAGAGAGAA GATAAGAACCATTTGTTATTGGAATTAGCAAAGATGGAGTTTAATACA TTGCAAGCTATCTATCAAGAAGAATTGAAGGAAATTTCTGGTTGGTGG AAAGATACTGGTTTGGGTGAAAAATTATCATTTGCAAGAAATAGATTA GTTGCTTCATTTTTGTGGTCAATGGGTATTGCATTTGAACCACAATTCG CTTACTGTAGAAGAGTTTTGACAATTTCTATTGCATTGATCACTGTTATT GATGATATATATGATGTTTACGGTACATTGGATGAATTAGAAATTTTTA CTGATGCTGTTGCAAGATGGGATATTAACTACGCTTTGAAGCATTTGCC AGGTTACATGAAAATGTGTTTCTTGGCATTGTACAACTTCGTTAACGAA TTTGCTTATTACGTTTTGAAACAACAAGATTTTGATATGTTATTGTCTAT TAAAAATGCTTGGTTGGGTTTAATCCAAGCATATTTGGTTGAGGCTAAG TGGTATCATTCTAAGTACACTCCAAAATTGGAAGAATACTTAGAAAAC 
GGTTTGGTTTCAATTACAGGTCCATTGATCATCGCTATTTCTTATTTGTC AGGTACTAACCCAATTATTAAGAAAGAATTGGAATTTTTAGAATCAAA TCCAGATATTGTTCATTGGTCTTCAAAGATTTTTAGATTACAAGATGAT TTGGGTACATCTTCAGATGAAATTCAAAGAGGTGACGTTCCAAAATCT ATTCAATGTTACATGCATGAAACTGGTGCATCAGAAGAAGTTGCTAGA GAACATATCAAGGATATGATGAGACAAATGTGGAAGAAAGTTAATGC ATACACAGCTGATAAAGATTCTCCATTGACTAGAACTACAACTGAATTT TTGTTGAATTTGGTTAGAATGTCACATTTCATGTATTTGCATGGTGACG GTCATGGTGTTCAAAACCAAGAAACAATTGATGTTGGTTTTACTTTGTT GTTCCAACCAATCCCATTAGAAGATAAGGATATGGCTTTTACTGCTTCT CCAGGTACTAAAGGTTAA

CltLS3 ATGATCAGAAGATCTGCTGATTATGGTCCAACAATTTGGTCATTCGATT ACATCCAATCTTTGGATTCAAAGTACAAGGGTGAATCTTACGCAAGAC AATTGGAAAAATTGAAGGAACAAGTTTCAGCTATGTTACAACAAGATA ATAAGGTTGTTGATTTGGACCCATTGCATCAATTAGAATTGATTGATAA TTTGCATAGATTAGGTGTTTCTTACCATTTCGAAGATGAAATTAAAAGA ACATTGGATAGAATACATAATAAGAATACTAATAAGTCTTTGTACGCT AGAGCATTGAAGTTTAGAATTTTGAGACAATACGGTTACAAGACACCA GTTAAGGAAACTTTTTCAAGATTCATGGATGAAAAGGGTTCTTTTAAAT TATCTTCACATTCAGATGAATGTAAAGGCATGTTAGCATTGTATGAAGC TGCATACTTGTTGGTTGAAGAAGAATCTTCAATTTTTAGAGATGCTATC AGATTCACTACAGCATATTTGAAGGAATGGGTTGCTAAGCATGATATT GATAAGAACGATAACGAATACTTGTGTACATTGGTTAAGCATGCATTA GAATTGCCATTGCATTGGAGAATGAGAAGATTAGAAGCTAGATGGTTC ATTGATGTTTACGAATCTGGTCCAGATATGAACCCAATCTTGTTAGAAT TGGCTAAGGTTGATTACAACATCGTTCAAGCAGTTCATCAAGAAGATTT GAAATACGTTTCAAGATGGTGGAAGAAAACTGGTTTGGGTGAAAAATT GAACTTCGCAAGAGATAGAGTTGTTGAAAATTTCTTTTGGACTGTTGGT GACATTTTCGAACCACAATTCGGTTATTGTAGAAGAATGTCTGCTATGG TTAACTGTTTGTTGACTTCAATTGATGATGTTTATGATGTTTACGGTACA TTGGATGAATTGGAATTGTTTACTGATGCTGTTGAAAGATGGGATGCA ACTACAACTGAACAATTGCCATACTACATGAAATTGTGTTTCCATGCAT TGTACAACTCTGTTAACGAAATGGGTTTTATTGCTTTGAGAGATCAAGA AGTTGGTATGATCATCCCATATTTGAAAAAGGCTTGGGCAGATCAGTG TAAGTCTTACTTAGTTGAGGCTAAGTGGTATAATTCAGGTTACATCCCA ACATTGCAAGAATACATGGAAAACGCTTGGATTTCTGTTACTGCACCA GTTATGTTGTTACATGCTTATGCTTTTACTGCTAACCCAATCACTAAGG AAGCATTGGAATTTTTACAAGATTCACCAGATATTATCAGAATTTCTTC AATGATTGTTAGATTGGAAGATGATTTGGGTACATCTTCAGATGAATTG AAGAGAGGTGACGTTCCAAAATCTATTCAATGTTACATGCATGAAACA GGTGTTTCAGAAGATGAAGCTAGAGAACATATCAGAGATTTGATCGCA GAAACTTGGATGAAAATGAACTCTGCTAGATTTGGTAATCCACCATAC TTACCAGATGTTTTTATTGGTATCGCTATGAATTTGGTTAGAATGTCTC 
AATGTATGTATTTGTACGGTGACGGTCATGGTGTTCAAGAAAACACTA AGGATAGAGTTTTGTCTTTGTTTATTGATCCAATTCCATAA

CstLS ATGGATAGAAGATCCGCTAATTACCAACCATCCATTTGGGATCATGATT TCTTGCAGTCTCTGAACTCTAACTACACTGACGAAACTTACAAAAGAA GGGCCGAAGAATTGAAAGGTAAGGTTAAGACTGCCATCAAGGATGTTA CTGAACCATTGGATCAGTTGGAATTGATCGACAACTTGCAAAGATTGG GTTTAGCCTACCATTTCGAACCAGAAATCAGAAACATCTTGAGGAACA TCCATAACCACAACAAGGACTACAATTGGCGTAAAGAAAACTTGTACG CTACCTCCTTGGAATTTCGTTTGTTGAGACAACATGGTTACCCAGTTTC TCAAGAAGTTTTCTCTGGTTTCAAGGATGATAAGGTCGGTTTCATCTGT GATGATTTCAAGGGTATCTTGTCATTGCATGAAGCCTCTTACTATTCCT TGGAAGGTGAATCTATTATGGAAGAAGCCTGGCAATTCACTAGCAAGC ACTTGAAAGAAATGATGATTACCTCCAACTCCAAAGAAGAGGACGTTT TTGTTGCTGAACAAGCTAAAAGAGCTTTGGAATTGCCATTGCATTGGA AAGCTCCAATGTTGGAAGCTAGATGGTTCATCCATGTTTACGAAAAGA GGGAAGATAAGAACCACTTGTTGTTGGAATTAGCCAAGTTGGAGTTTA ACACCTTGCAAGCTATCTACCAAGAAGAGTTGAAGGATATTTCAGGTT GGTGGAAAGATACTGGTTTGGGTGAAAAATTGTCCTTCGCTAGAAATA GATTGGTCGCTTCTTTTTTGTGGTCTATGGGTATTGCTTTCGAACCTCAA TTTGCCTACTGTAGAAGAGTTTTGACCATTTCCATTGCCTTGATCACCG TTATCGATGATATCTACGATGTCTACGGTACTTTGGACGAATTGGAAAT TTTCACTGATGCTGTTGCTAGATGGGATATTAACTACGCCTTGAAACAT TTGCCAGGTTACATGAAGATGTGTTTCTTGGCCTTGTACAACTTTGTCA ATGAGTTCGCTTACTACGTCTTGAAGCAACAAGATTTCGACATGCTGTT GTCTATCAAACATGCTTGGTTGGGTTTGATTCAAGCCTATTTGGTTGAA GCCAAGTGGTATCATTCTAAGTACACCCCAAAACTGGAAGAGTACTTG GAAAACGGTCTGGTTTCTATTACTGGTCCATTGATTATCACCATCAGCT ACTTGTCTGGTACGAACCCAATTATCAAGAAAGAGCTGGAATTTTTGG AGAGCAACCCAGATATAGTTCACTGGTCCTCTAAGATTTTCAGATTGCA AGATGACTTGGGCACTTCCTCTGATGAGATTCAAAGAGGTGACGTTCC AAAGTCTATTCAATGTTACATGCACGAAACTGGTGCCTCTGAAGAAGT TGCTAGAGAACATATTAAGGACATGATGAGACAGATGTGGAAGAAGG TTAACGCTTATACTGCTGATAAGGATTCTCCATTGACTAGAACTACCGC CGAATTTTTGTTGAACTTGGTTAGAATGTCCCACTTCATGTACTTGCAT GGTGATGGTCATGGTGTCCAAAATCAAGAAACTATCGATGTTGGTTTC ACCTTGTTGTTCCAACCTATTCCATTAGAGGATAAGGATATGGCTTTTA CTGCTTCTCCAGGTACTAAGGGTTAA

$P t t L S$ ATGCAAAGAAGATCCGGTAACTACTCTCCATCCTTCTGGAACGCTGACT ACATTTTGTCTTTGAACAACCACTACAAGGAAGAATCTCGTCACATGA AGAGAGCTGGTGAATTGATTGTTCAAGTTAAGATGGTTATGGGTAAGG AAACTGATCCAGTTGTTCAATTGGAATTGATTGATGACTTGCACAAGTT GGCTTTGTCTCACCACTTCGAAAAGGAAATCAAGGAAATCTTGTTCAA CATCTCTATCTACGATCACAAGATCATGGTTGAAAGAGATTTGTACTCT ACTGCTTTGGCTTTCAGATTGTTGAGACAATACGGTTTCAAGGTTCCAC 
AAGAAGTTTTCGACTGTTTCAAGAACGACAACGGTGAATTTAAGAGAT CGTTGTCTTCTGACACCAAGGGTTTGTTGCAATTGTACGAAGCTAGTTT CTTGTTGACTGAAGGTGAAATGACTTTGGAATTGGCTAGAGAATTTGCC ACCATCTTCTTGCAAGAAAAGTTGAACGACAAGACTATCGATGATGAT GATGATGCTGACACTAACTTGATCTCTTGCGTTAGACACAGCTTGGACA TCCCAATACACTGGAGAATACAAAGACCAAACGCTTCTTGGTGGATTG ATGCTTACAAGAGAAGATCGCACATGAACCCATTGGTTTTGGAATTGG CCAAGCTCGACTTGAACATCTTCCAAGCGCAATTCCAACAAGAATTGA AGCAAGACTTGGGTTGGTGGAAGAACACTTGTTTGGCTGAAAAGTTGC CATTCACTAGAGATAGATTGGTTGAATGTTACTTCTGGTGTACTGGTAT CATTCAACCATTGCAACACGAAAACGCTAGAGTTACTTTGGCCAAGGT TAACGCTTTGATCACCACTTTGGATGATATTTACGATGTCTACGGTACT TTGGAAGAATTGGAATTGTTCACCGAAGCTATTAGAAGATGGGATGTT TCTTCTATTGACCACTTGCCAAACTACATGCAATTGTGTTTCTTGGCTTT GAACAACTTCGTCGATGACACTGCTTACGATGTCATGAAGGAAAAGGA TATCAACATCATCCCATACTTGAGAAAGTCTTGGTTGGATTTGGCTGAA ACTTACTTGGTTGAAGCTAAGTGGTTCTACTCTGGTCACAAGCCAAACA TGGAAGAATACTTGAACAACGCTTGGATTTCTATTTCTGGTCCAGTTAT GTTGTGTCACGTTTTCTTCAGAGTTACTGATTCTATTACTAGAGAAACT GTTGAATCTTTGTTCAAGTACCACGATTTGATTAGATACTCTTCTACCA TTTTGAGATTGGCTGATGATTTGGGTACTTCTCTCGAAGAAGTAAGCAG AGGTGATGTTCCAAAGTCTATTCAATGTTACATGAACGACAACAACGC TTCTGAAGAAGAAGCTAGAAGACACGTCAGATGGTTGATCGCTGAAAC TTGGAAGAAGATTAACGAAGAAGTTTGGTCTGCTGATTCTCCATTCTGT AAGGATTTCATCGCTTGTGCTGCTGATATGGGTAGAATGGCTCAATTCA TGTACCACAACGGTGATGGTCACGGTATCCAAAACCCACAAATCCACC AACAAATGACTGACATCTTGTTCGAACAATGGTTGTAA

ShtLS ATGTGCAGCCACAGTACCACTTCATCAATGGATGGTTTTGAAGATGCA AGGGATAGAATAAGGGAAAATTTTGGGAAATTAGAGTTATCACCTTCT TCCTATGACACAGCATGGGTAGCTATGGTCCCTTCAAAACATTCACTAA ATGAGCCATGTTTTCCACAATGTTTGGATTGGATTATTGAAAATCAAAG AGAAGATGGATCTTGGGGACTAAACCCTACCCATCCATTGCTTCTAAA GGACTCACTTTCTTCCACTCTTGCATGTTTGCTTGCACTAACCAAATGG AGAGTTGGAGAGGAGCAAATCAAAAGAGGTGTTGGCTTCATTGAAACG TATGGTTGGGCAGTAGATAACAAGGATCAAATTTCACCTCTAGGATTT GAAGTTATATTTTCTAGTATGATCAAATCTGCAGAGAAATTAGATTTAA ATTTGCCTTTGAATCTTCATCTTGTAAATTTGGTGAAATGCAAAAGAGA TTCAACAATTAAAAGGAATGTTGAATATATGGGTGAAGGAGTTGGTGA ATTATGTGATTGGAAGGAAATAATAAAGTTACATCAAAGACAAAATGG TTCATTATTTGATTCACCAGCCACTACTGCAGCTGCCTTGATTTATCATC AACATGATAAAAAATGCTATCAATATCTTAATTCAATCTTGCAACAAC ACAAAAATTGGGTTCCCACTATGTATCCAACAAAGATACATTCATTGCT TTGCTTGGTTGATACACTTCAAAATCTTGGAGTACATCGGCATTTTAAA TCAGAAATAAAGAAAGCTCTAGATGAAATATACAGGCTATGGCAACAA 
AAGAATGAAGATAATTTCTCAAATGTCACCCATTGTGCTATGGCTTTTC GACTTCTAAGGATGAGCTACTATGATGTCTCCTCAGATGAACTAGCAG AATTTGTGGATGAAGAACATTTCTTTTCAACAAGTGGGAAATATACAA GTCATGTTGAAATTCTTGAACTCCACAAAGCATCACAATTGGCTATTGA TCATGAGAAAGATGACATTTTGGATAAGATTAACAATTGGACAAGAAC TTTTATGGAGCAAAAACTCTTAAACAATGGCTTCATAGATAGGATGTC AAAGAAAGAGGTGGAACTTGCTTTGAGGAAGTTTTATACCACATCTCA TCTAGCAGAAAATAGAAGATATATAAAGTCATACGAAGAGAACAATTT TAAAATCTTAAAAGCAGCTTATAGGTCACCCAACATTAACAATAAGGA CTTGTTAGCATTTTCAATACACGACTTTGAATTATGCCAGGCTCAACAC CGAGAAGAACTTCAACAACTCAGGAGGTGGTCTGAAGATTATAGATTG GACCAACTCGGACTTTTAGAACGATATATATATGCTAGTTACTTAATTG GTGTTATTGTTATCCCCGAGCCTGAATTATCCGATGCTCGTCTCATGTA CACGAAATACATCATGCTCCTGACTATTGTCGATGATCATTTCGATAGT TTTGCATCTAAAGATGAATGTCTCAACATCATTGAATTAGTAGAAAGGT GGGATGACTATGCAAGTGTAGGTTATAAATCTGAGAAGGTTAAAGTTT TTTTTTCTGTTTTCTATAAATCAATAGAGGAGCTTGCAACAATTGCTGA AATTAAACAAGGACGATCTGTCAAAAATCACCTTATTAATTTGTGGCTT GAATTGATGAAGTTGATGTTGATGGAACAAGTAGAGTGGTGTTCTGGC AAGACAATACCAAGCATAGAAGAGTATTTGTATGTTACATCTGTAACA TTTGGTTCAAAATTGCTTCCTCTCTCAACACAATATTTTCTTGGAATAA AAATATCCAAAGATCTTTTAGAAAGTGATGAAATAGATGGTTTATGCA ATTGTAGCGGTAGAGTCATGCGAATCCTTAATGATTTACAAGATTCCAA GAAAGAACAAAAGGAGGGCTCAATAACTTTAGTCACATTACTAATGAA AAGTATGTCTGAGGAAGAAGCTATAATGAAGACAAAGGAAATCTTGG AAATGAATAGAAGAGAGTTGTTGAAAATGGTTTTAGTTCAAAAAAAGG GAAGCCAATTGCCTCAATTATGCAAAGATATATTTTGGAGGTCAAGCA AATGGGCTGATTTCACTTATTCACAAACTGATGCATATAGAATTCCAGA GGAAATGAAGAATCACATTGATGAAGTCTTTTACAAACCACTCAATCA TTAA

ArtLS ATGGAAAGAAGATCGGGTAACTACTCTCCATCCAGATGGGATGTCGAT TTCATTCAATCTTTGAACTCTGATTACCAAGAAGAAAGACACACTAGA AGAGCTTCTGAATTGATTACTCAAGTTAAGATGTTGATGGAAAAGGAA ACTACCGATCCAATTAGACAATTGGAATTGATCGACGACTTGCAAAGA TTGGGTTTGTCTGATCACTTCCAAAACGAATTTAAGGAAATCTTGAACA CTATCTACTTGGACAACAAGTACTACAACATTAACATTATGAGAGAAG AATCTCGTGATTTGTACTCTACTGCTTTGGCTTTCAGATTGTTGAGAGA ACACGGTTTCCAAGTTGCTCAAGAAGTTTTCGAATGTTTCAAGAACGA AGAAGGTGATTTCAAGGCTTCTTTGATCGACGACACCAGAGGTTTGTTG CAATTGTACGAAGCTAGTTTCTTGTTCAAGGAAGGTGAAAACACTTTG GAAATCGCTAGAGAATTTACCACCAAGATTTTGCAAGAAAAGTTGAAG GGTGATGAAATTGATGACAACTTGTTGTCTTCCATTAGATACTCTTTGG AAATCCCAAACTACTGGTCTGTTGTTAGACCAAACGTTTCTGTTTGGAT TGATGAATACAGAAAGAGATCGGACATGAACCCAGTTGTTTTGGAATT 
GGCCATCTTGGACGCTAACATTGTTCAAGCTCAATTGCAATTGGAATTG AAGGAATCTTTGAGATGGTGGAGAAACACTTGTTTCGTTGAAAAGTTG CCATTCGCTAGAGATAGATTGATCGAATCTTACTTCTGGTCTACTGGTA TGGTTGAACCAAGACAACACGCTAACGCTAGAATCATTATGGCCAAGG TTATCGCTTTGATCACTGTCATGGATGATATTTACGATGTCTACGGTAC TTTGGAAGAATTGGAACAATTCACTGAAGCCTTCCGTCGTTGGGATGTG AGCTCTATCGACCAACTCCCAACTTACATGCAATTGTGCTTCCTGGCTA TCAACAACTTCGTTGACGACACTGCTTACAACGTTTTGAAGGAATCTGG TGTCAACGTTATGACTTACTTGAGAAAGTCTTGGGTTGATCAAGCTGAA AACTACTTGATGGAATCTAAGTGGTACTACTCTGGTCACAAGCCATCTT TGGAAGAATACTTGGAAAACTCTTGGATCTCTGTCTCTGGTCCATGTGT TTTGACCCACGAATTTTTCGGCGTTACTGATAGCTTGGCTAAGGACACT CTCGACAGCTTGTACGAATACCATGATATCGTTAGATGGTCCTCTTACT TGTTGAGATTGGCTGATGATTTGGGTACTTCCGTTGAAGAAGTTTCTCG TGGTGACGTTCCAAAGTCTATCCAATGTTACATGCACGACAACGATGCT TCTGAAGAAGAAGCTAGACAACACATCAAGGGTTTGATCAGAGAAATG TGGAAGAAGATGAACGTTGAAAGAGTTTCTGAAGATTCTCCATTCTGT AGAGATTTCATCAGATGTTGTGAAGATTTGGGTAGAATGGCTCAATTC ATGTACCACTACGGTGATGGTCACGGTACTCAACACCCAAAGATCCAC CAACAAATCGCCGCTTGTTTGTTCCAACCATTCGCTTAA

SINDPS1 ATGTCTGCTAGAGGTTTGAACAAGATTTCGTGCAGCTTGAACTTGCAAA CTGAAAAGTTGTGTTACGAAGATAACGATAACGATTTGGATGAAGAAT TGATGCCAAAGCACATTGCTTTGATCATGGATGGTAACAGAAGATGGG CTAAGGATAAGGGTTTGGAAGTTTACGAAGGTCACAAGCACATTATTC CAAAGTTGAAGGAAATTTGTGACATTTCTTCTAAGTTGGGTATCCAAAT TATCACTGCTTTCGCTTTCTCTACTGAAAACTGGAAGAGATCCAAGGAA GAAGTTGATTTCTTGTTGCAAATGTTCGAAGAAATCTACGATGAATTTT CTCGTTCGGGTGTTAGAGTTTCTATTATCGGTTGTAAGTCCGACTTGCC AATGACTTTGCAAAAGTGTATCGCTTTGACTGAAGAAACTACTAAGGG TAACAAGGGTTTGCACTTGGTTATTGCTTTGAACTACGGTGGTTACTAC GACATCTTGCAAGCTACTAAGTCTATTGTTAACAAGGCTATGAACGGTT TGTTGGATGTTGAAGATATCAACAAGAACTTGTTCGATCAAGAATTGG AATCTAAGTGTCCAAACCCAGATTTGTTGATCAGAACTGGTGGTGAAC AAAGAGTTTCTAACTTCTTGTTGTGGCAATTGGCTTACACTGAATTTTA CTTCACCAACACTTTGTTCCCAGATTTCGGTGAAGAAGATTTGAAGGAA GCTATCATGAACTTCCAACAAAGACACAGAAGATTCGGTGGTCACACT TACTAA 



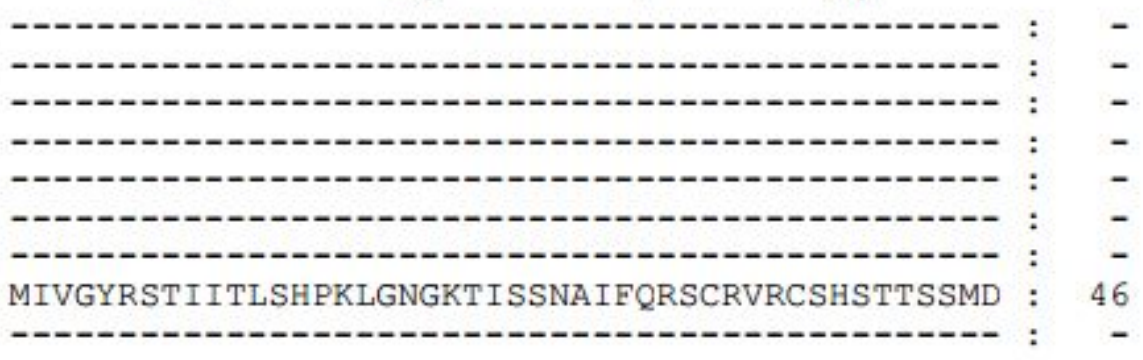

CuLs1 :

*

60

*

80

CuLs2

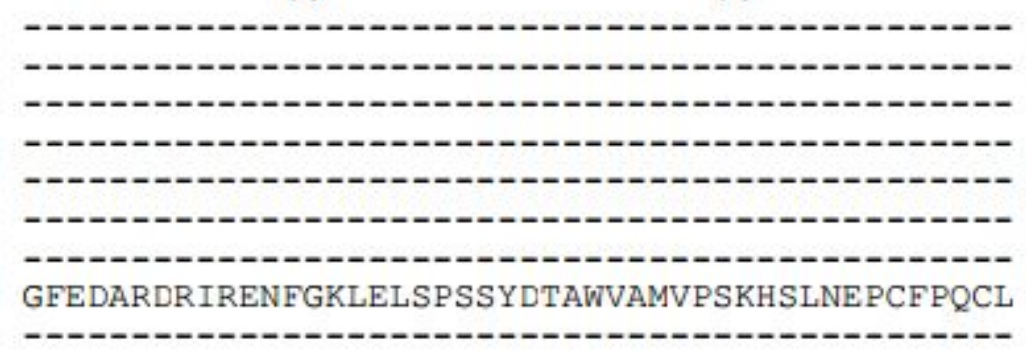

CILS2

CILS3

CsLS

PtLS

SILS

ArLS

CuLS1:
CuLS2 :
ClLS1 :
ClLS2 :
ClLS3 $:-$
CsLS :
PtLS :
SILS :
ArLS :

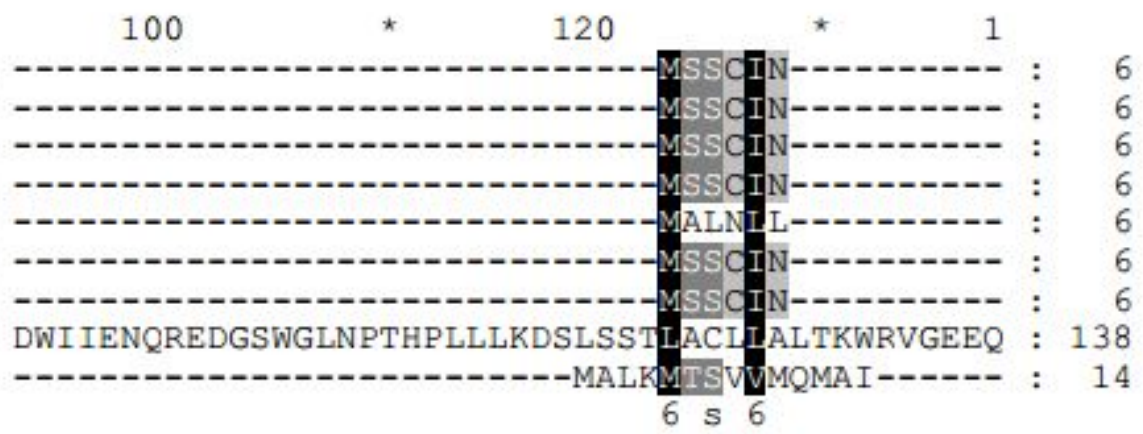

CuLs1:
CuLS2 :
ClLS1:
ClLS2 :
ClLS3 :
CsLS :
PtLS :
SILS :
ArLS :

40

160

180

-- - - PSTIATSVN---GEKCIPIATNRAAIRIMAKNK----PV : 38

-- 38

------PSTIVTSVN---AEKCIPIATNKAAIRIMAKYK----PV : 38

------PSTIVTSAN---GEKCLPIATNKAAIRIMAKNK----PV : 38

$------S S I$ PAACN----ETRISIPLSSKVNGFVPPITQVQYPM : 40

-- - - PSTIATSVN---GEKCIPLATNRAAIRIMAKNK----PV : 38

- - - PSTIATSVN---GEKYL PLATNRAAIRITAKNK----PV : 38

IKRGVGFIEIYGWAVDNKDQISPIGFEVI FSSMIKSAEKLDLNLPI : 184

-----PTKIANFVHNSDNNESKIVRNVSTTCTSAATPRLRLPV : 53

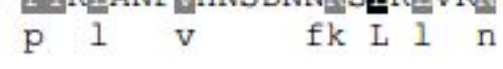




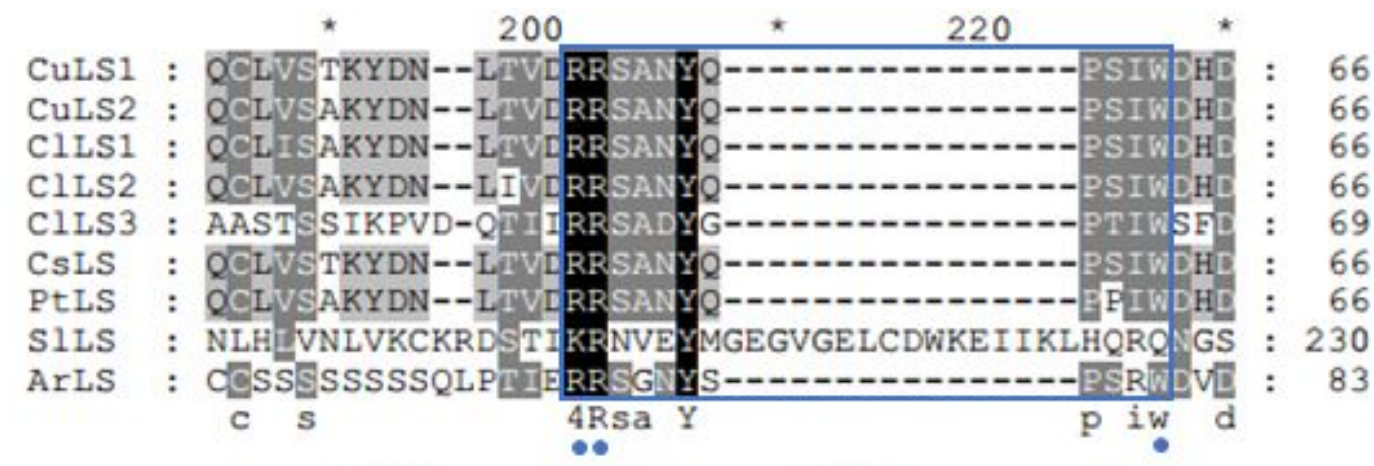

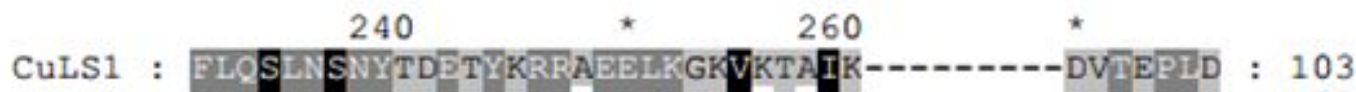

CULS2 : FLOSLNSNYTDETYKRREEILKGKVMTTIK--------DVREPLN : 103

CILS1 : FLQSLNSNYTDEAYKRRABDLRGKVKIAIK--------DVIEPID : 103

ClLS2 : FLCSLNSNYTDDTYRRRABELKGKVKIAIK---------DVTEPID : 103

C1LS3 : YIQSLDSKYKGSSYARQLELEECVSAMLQQDN----KVVDLDPIH : 111

CSLS : FLOSLNSNYTDDTYKRFAESLKGKVKTAIK--------DVTEPID : 103

PLLS : FLOSLNSPYTDETYRREADELKGKVKTAIE---------DVTEPID : 103

SILS : LFDSPATTAAALI HQHDKKCYQYLNSILQQHKNWVPTMYPIKIHS : 276

ArLS : FIOSUNSDYQEDRHTRAAS DIITIVVKMIMEK-------ETMDPIR : 121 qSi 3 y e y rr eel $6 \quad 6 \quad$ t p

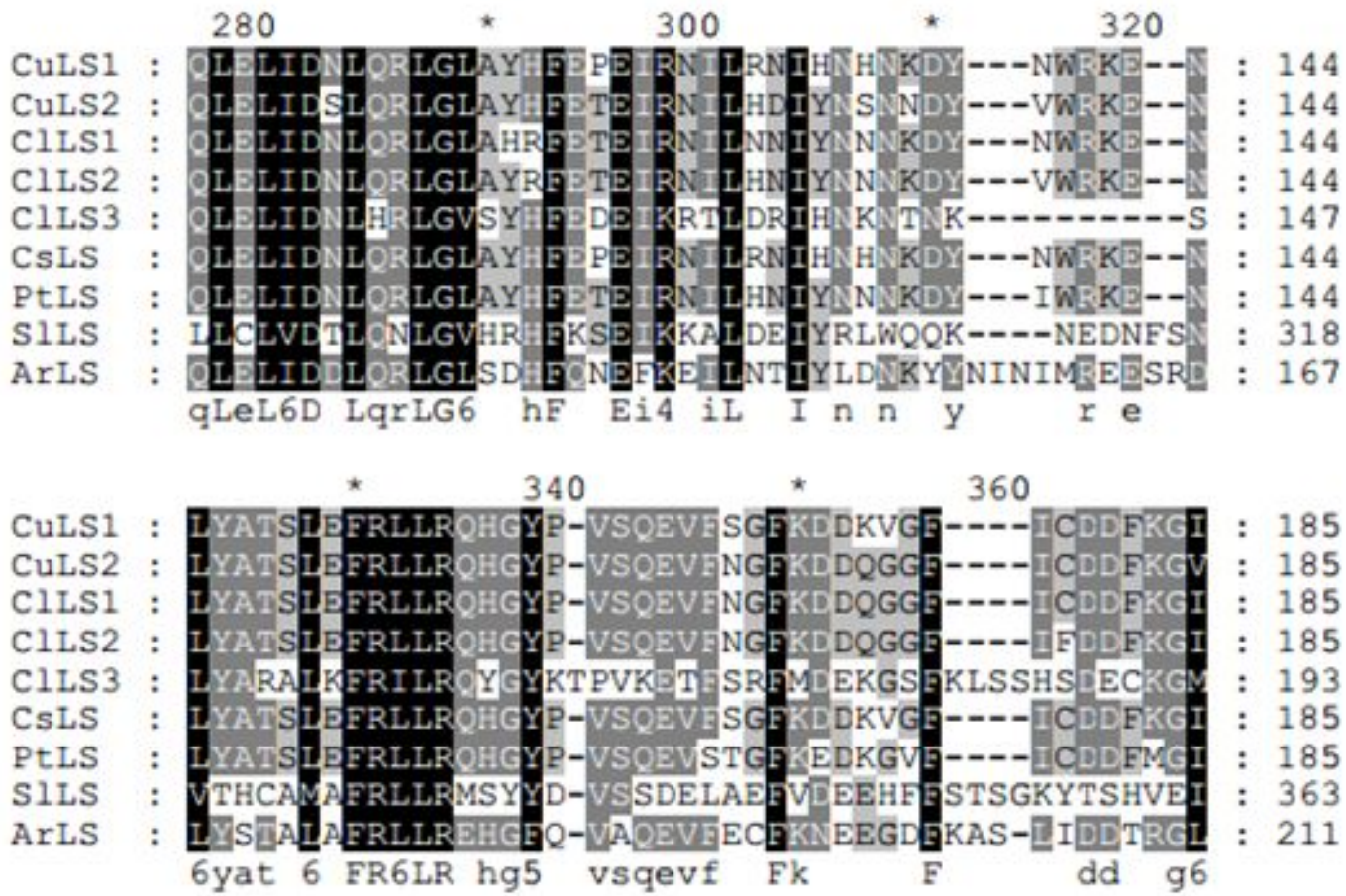




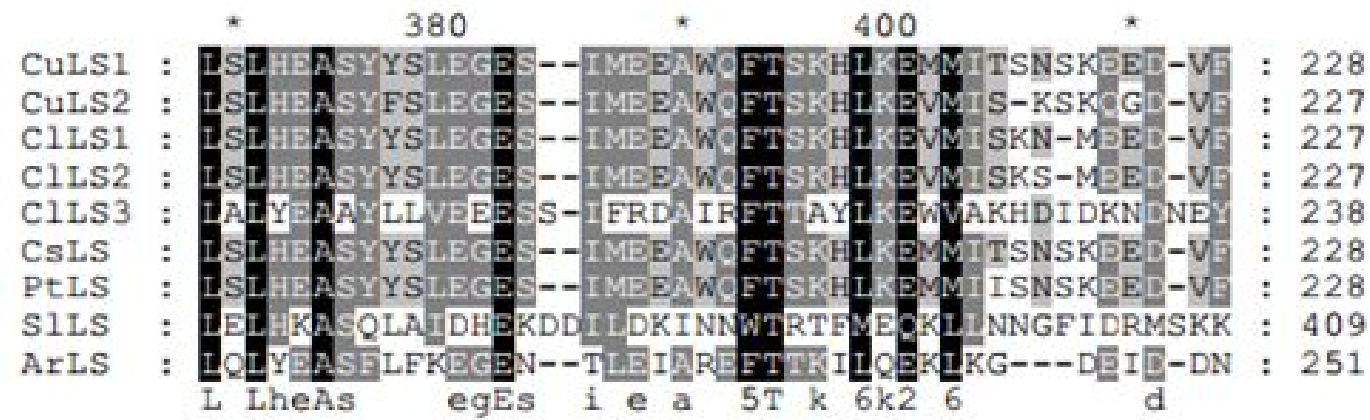

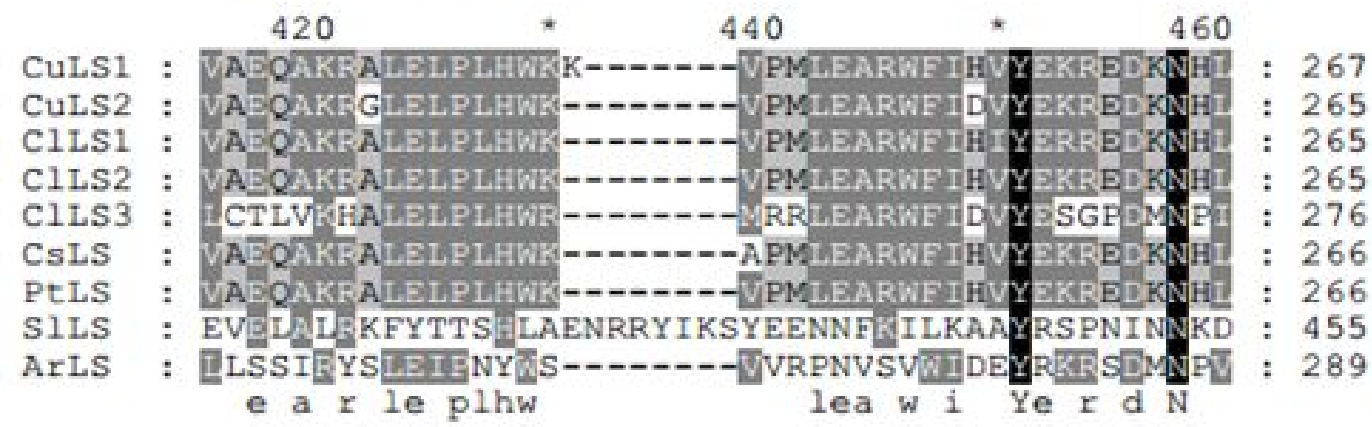

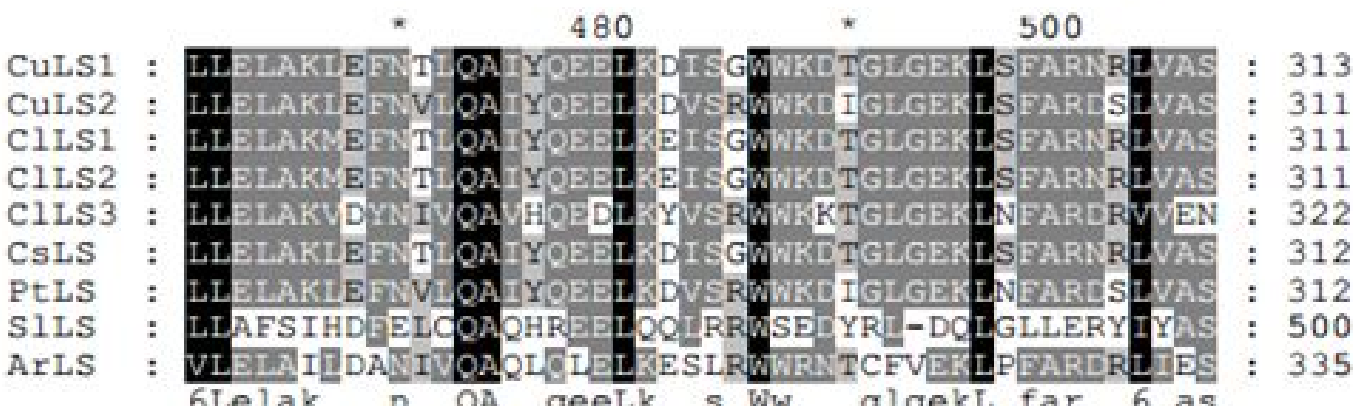

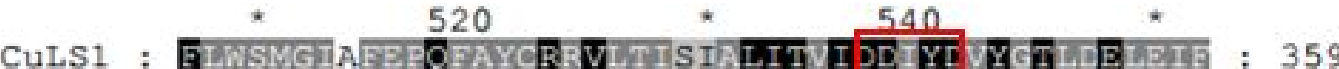

CuLS2 : FVWSMGIVPER QPAYCRA ILTITFALISVI DD YLVYGTLEELELE : 357

CILS1 : GLWSMGIAFIPQEAYCRRVLTISIALITVI DDIYDVYGTLDELEIF : 357

CILS2 : FLWSMGIAFERQFAYCRRVLTISIALITVI DDIYDVYGTLDELEIE : 357

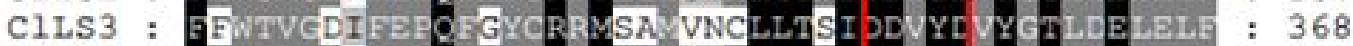

CSLS : GLWSMGIAEERQFAYCRRVLTISIALITVI DDIYDVYGTLDELEIF : 358

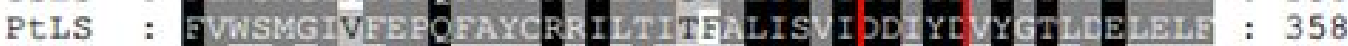

SILS : YLIGUIVIPERELSDARIMYGKYIMLLT IVPDHFLSEASKDECLNI : 546

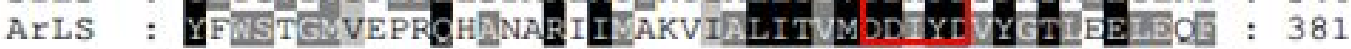

$5 \mathrm{w} g$ fep2faycRr6 t aL63 6DD 5Dv5g3idEle $f$ 


\section{0}

*

580

CuLS1 : TDAVARWDINYALKHLEGYMKCFLALYNFVNEFAYYVLKQODFDM : 405
CuLS2 : ADAVERWDINYALNHLBDYMKICFLALYNIVNEFTYYVLKQODFDI : 403

CILS1 : TDAVERWDINYALKHLPGYMNCFLALYNFVNEFAYYVLKQQDFDI : 403

CILS2 : TDAVARWDINYALKHLBGMINCFLALYNFVNDFAYYVLKOQDFDM : 403

CILS3 : TDAVERWDAT-TTEQLEYYMKCFHALYNSVNEMGEIALRDOEVGM : 413

CSLS : TDAVARWDINYALKHLEGMKNELALYNFVNEFAYYVLKOQDFDM : 404

PELS : ADAVERWDINYALNHLEDYMKICFLALYNIVNEFTYYVLKQODFDI : 404

SILS : IELVERWDDYASVGYKSEKVKVFFSVFYKSIEDLATIAEIKOGRSV : 592

ArLS : TEMFRRWDVS-SIDQLETYMQLCFLAINNFVDDTAYNVLKESGVNV : 426 dav RWD Ip y6k6cFla yn 6 e vl q 6

$00 \quad$ * 620 * 640

CuLS1 : IL-SIKHĀTGLIQAYLVEA-KhYHSKYTRKLEYLENGIVSITGE : 449

CuLS2 : IR-SIKNAWIRNIOAYLVEA-KWYHGKYTFTLGEFLENGIVSIGGE : 447

CILS1 : IL-SIKNAWLGLIQAYLVEA-KWYHSKYTHKLEYLENGLVSITGE: 447

C1LS2 : LL-SIKNAWLGLIOAYLVEA-KWYHSKYTEKLEYLENGIVSITGE : 447

CILS3 : IIPYLKKAnADQCKSYLVEA-KWYNSGYIFTLOEYMENAWISVTAR : 458

CSLS : LL-SIKHAWLGLIOAYLVEA-KWYHSKYTHKLEEYLNGLVSITGE : 448

PtLS : IR-SIKNAWIRNIQAYLVEA-KWYHGKYTFTLGELENGIVSIGGE : 448

SILS : KN-HLINLWhLELIKLMLMEQVEhCSGKTIFSIEEYLYVTSVTFGSK : 637

ArLS : МT-YLRSWVDQAENYLMES- KWYYSGHKESLDEYLNSWISVSGE : 470 $6 \mathrm{aW} \quad \mathrm{yL} 6 \mathrm{Ea}$ kWy ky P 6 E56en 63 gP

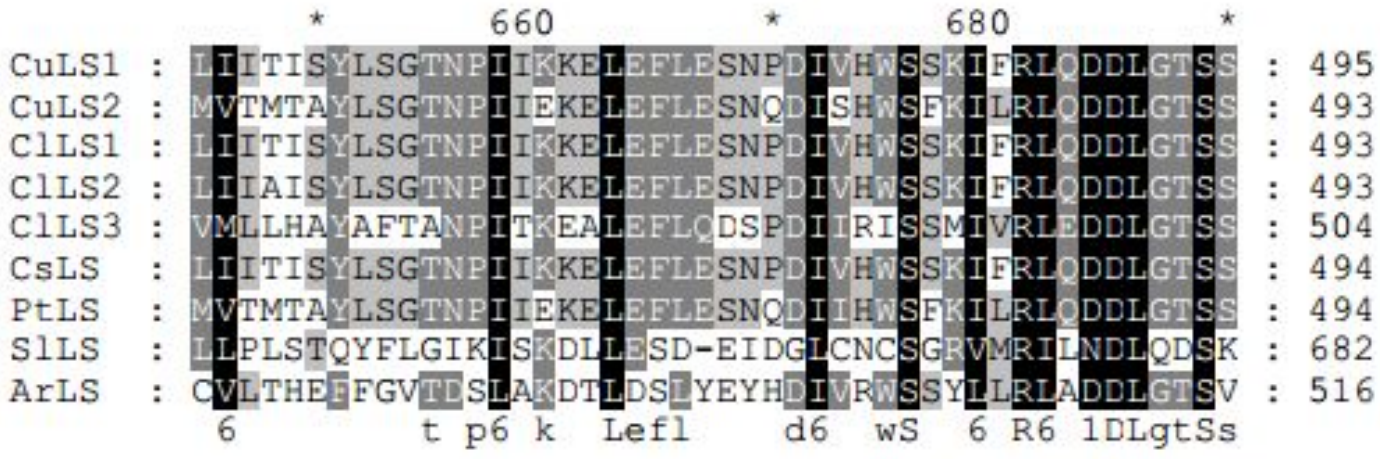
$700 \quad * \quad 720$

CuLS1 : DEIQRGDVPKSIQCYMHETGASEDVARBHIKDMMRQMWKKVNAYTA : 541

CuLS2 : DEIRRGDVPKSIQCYMHETGASEDVARBH IKDMMRQMWKKVNAYRA : 539

CILS1 : DEIQRGDVPKSIQCYMHETGASEEVARQH IKDMMRQMWKKVNAYTA : 539

CILS2 : DEIQRGDVPKSIQCYMHETGASEEVAREHIKDMMRQMWKKVNAYTA : 539

CILS3 : DELKRGDVPKSIQCYMHETGVSEDEAREH IRDIIAETWMKMNSARF : 550

CSLS : DEIQRGDVRSIQCYMHETGASEDVARBH IKDMMRQMUKKVNAYTA : 540

PLLS : DEIORGEVRKSIQCYMHETGASEDVARDH IKDMMROMWKKVNAYRA : 540

SILS : KEQKEGSITLVTLLMKSMSEEEA IMKTKEILEMNRRELLKMVLVQK : 728

ArLS : EEVSRGDVPKS IOCYMHDNDASEEEAROH IKGLIREMWKKMNVERV : 562 dE rGd6pksiqcymhe gasee ar hI d6 r mwkK6n 


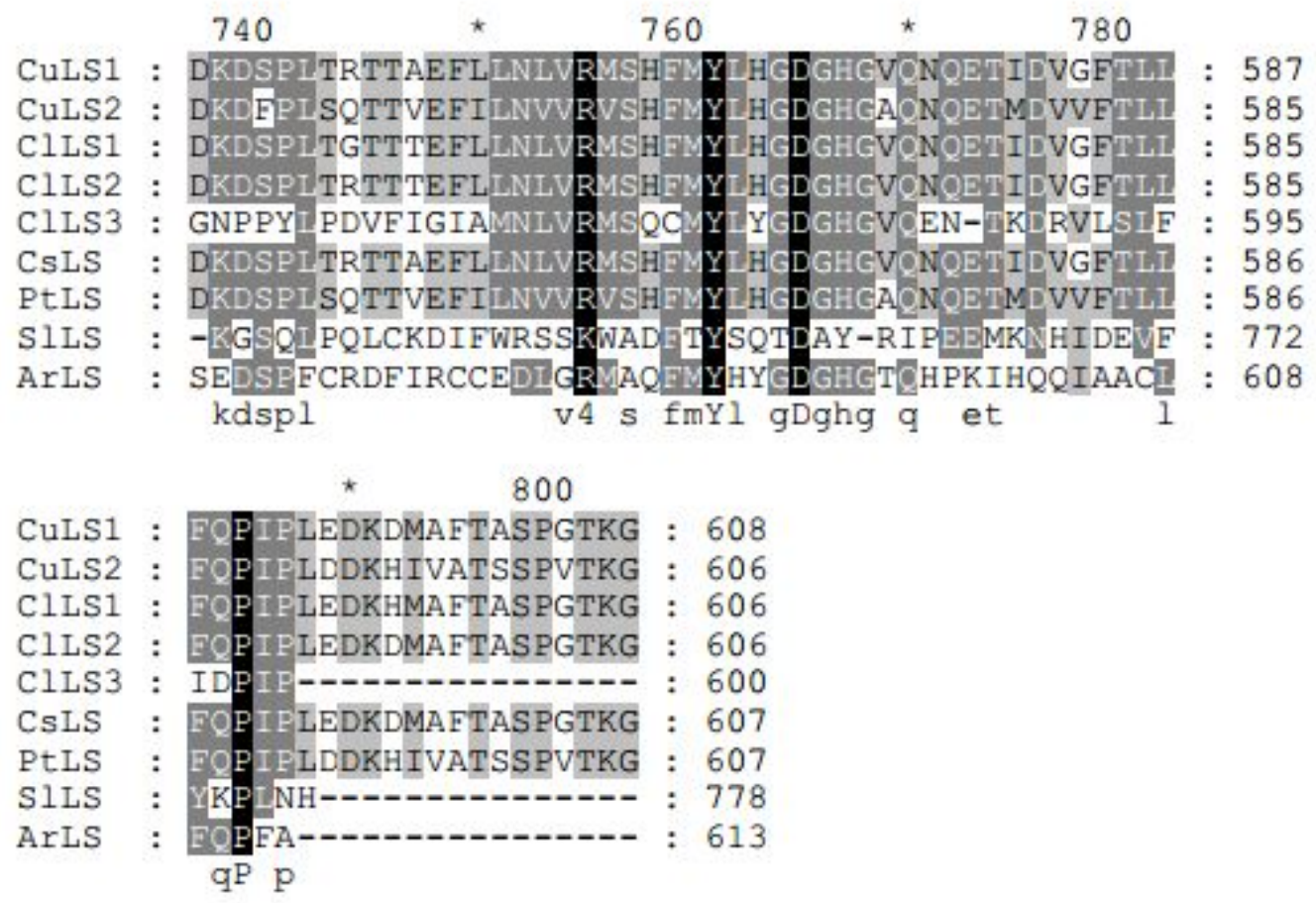

Fig. S1. Alignment of amino acid sequences of nine limonene synthases. Blue box indicates the RRX8W motif. Red box indicates the DDXXD motif.

CuLS1 (GenBank: AB110637.1), CuLS2 (GenBank: AB110636.1), ClLS1 (GenBank: AF514287.1), ClLS2 (GenBank: AF514289.1), ClLS3 (GenBank: AF514286.1), CsLS (GenBank: KU746814.2), ArLS (GenBank: AY055214.1), ShLS (GenBank: JN990693.1). 


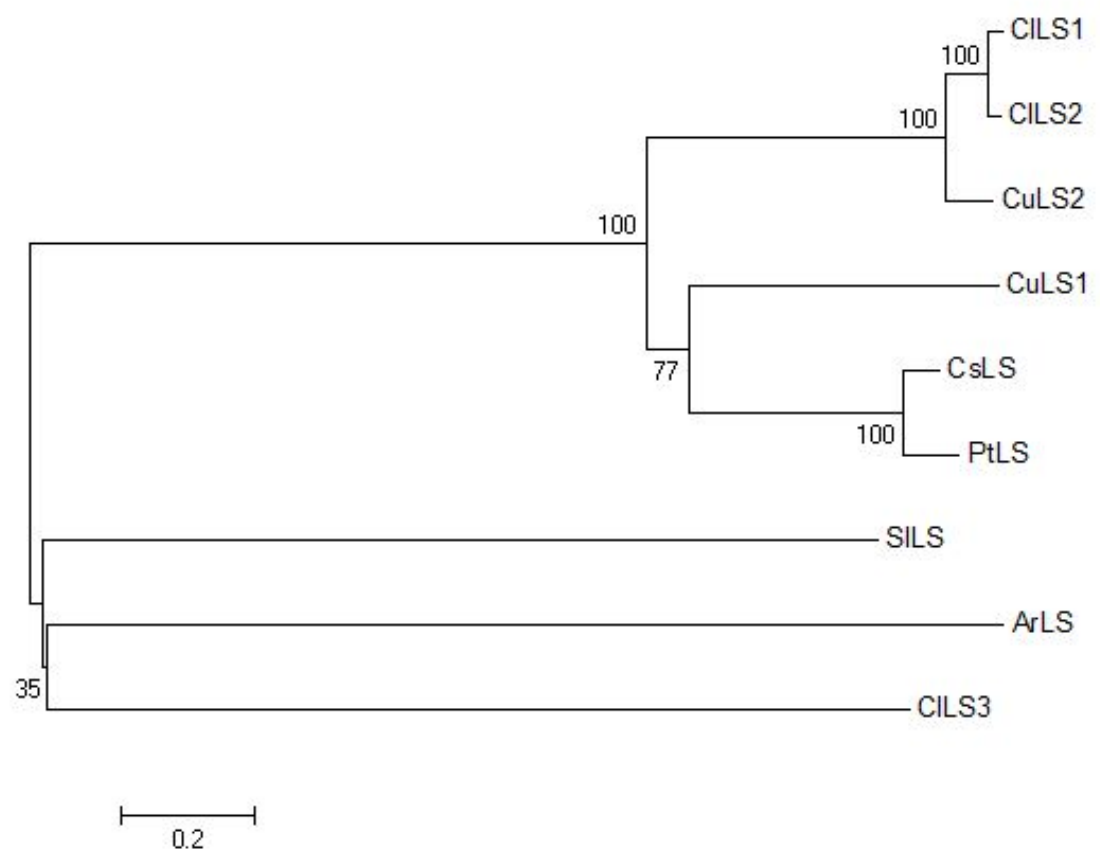

Fig. S2. Phylogenetic analysis of nine LSs proteins by using the neighbor-joining method. 


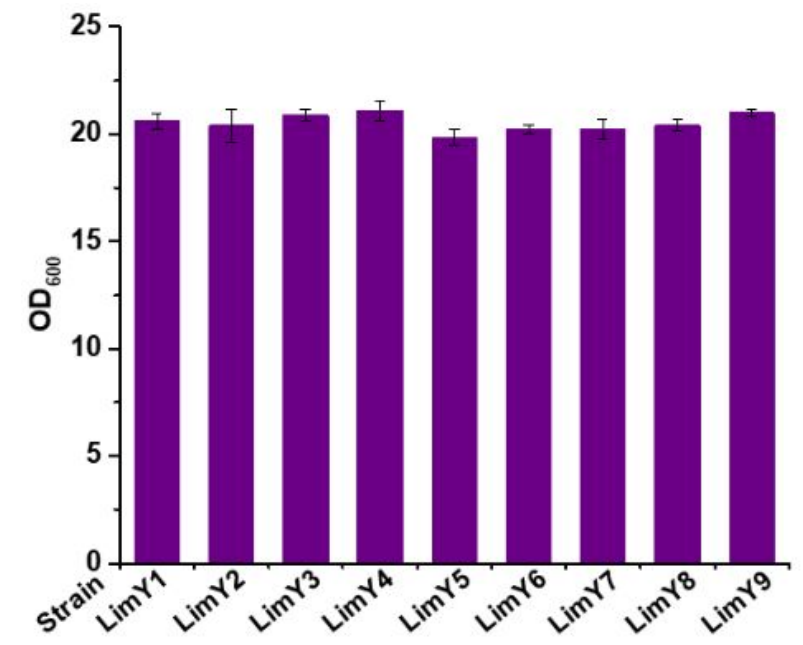

Fig. S3. Biomass of limonene-producing strains harboring the CLB pathway at $48 \mathrm{~h}$.

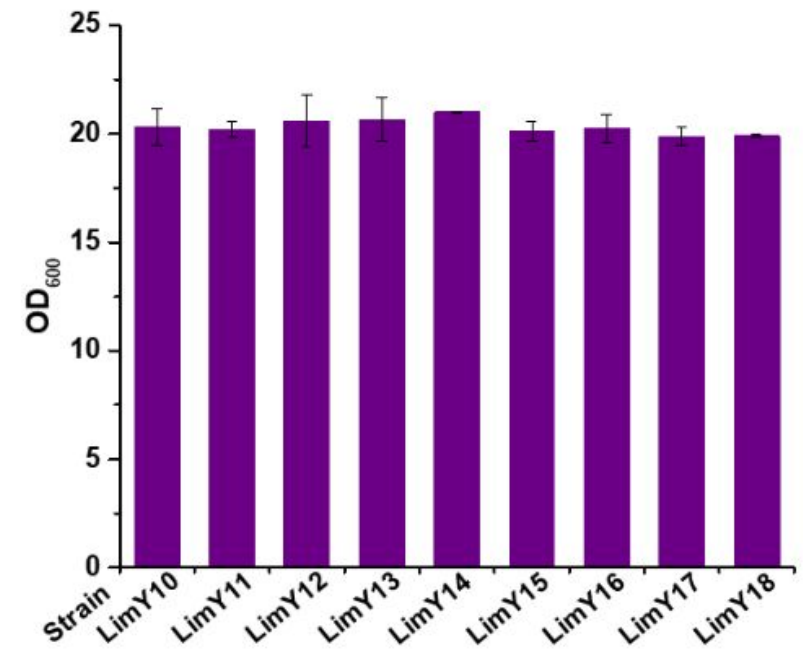

Fig. S4. Biomass of limonene-producing strains harboring the OLB pathway at $48 \mathrm{~h}$. 


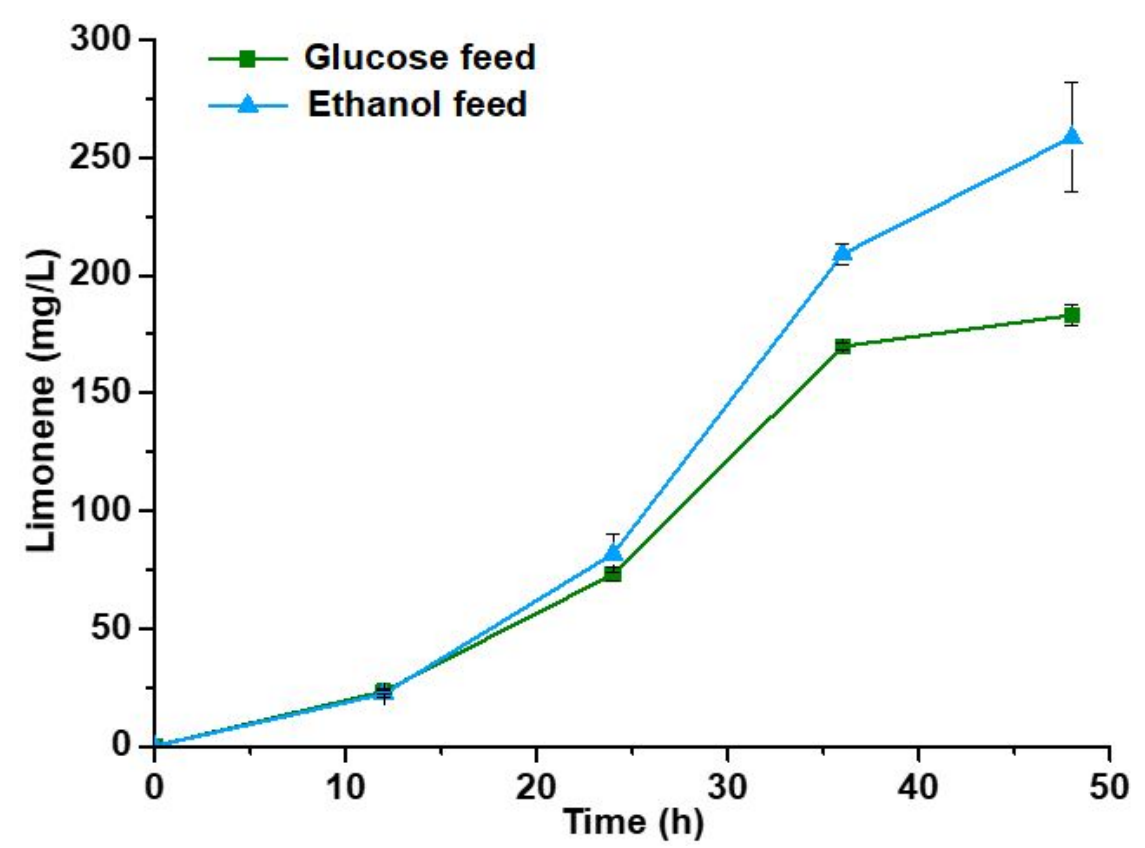

Fig. S5. Titer of limonene in strain LimY28 feeding with glucose and ethanol at $20 \mathrm{~h}$, respectively. 